\section{TÉRMINOS DE GÉNERO NATURAL E IDENTIDADES TEÓRICAS}

\author{
Luis Fernández Moreno \\ UCM, España
}

\section{TERMS OF NATURAL KIND AND THEORETICAL IDENTITIES}

RESUMEN: Kripke afirma en Naming and Necessity que existen ciertas similitudes entre los nombres propios y los términos de género natural. Una de ellas radica en que ambos tipos de expresiones figuran en enunciados de identidad que, en caso de ser verdaderos, son necesarios a posteriori; en el caso de los términos de género natural Kripke denomina a estos enunciados "identidades teóricas". Kripke sostiene que esta similitud se sigue de otra, consistente en que ambos tipos de expresiones son designadores rígidos. Mi objetivo en este artículo es alegar que, aunque cabe sostener que los términos de género natural son designadores rígidos, esta propiedad de los términos de género natural no permite justificar el rasgo de necesidad que Kripke atribuye a las identidades teóricas. A este respecto centraré mis consideraciones en un tipo de términos de género natural, los términos de sustancias químicas, a los que aludiré como "términos de sustancia".

PALABRAS CLAVE: Término de género natural; identidad teórica; necesidad; designador rígido.

teóricas" o "identidades teóricas" -en lo siguiente optaré por esta última denominación- y pone como ejemplos de tales enunciados "El agua es $\mathrm{H}_{2} \mathrm{O}$ " y "El oro es el elemento con el número atómico 79". Ahora bien, Kripke sostiene que esta similitud entre los términos de género natural y los nombres propios se sigue de otra, consistente en que los términos de género natural son, como los nombres propios, designadores rígidos. Kripke afirma:

Las identidades teóricas, de acuerdo con la posición que defiendo, son [...] identidades que involucran dos designadores rígidos y, por tanto, son ejemplos de lo necesario a posteriori (Kripke, 1980, 40).

Mi objetivo en este artículo es alegar que, aunque cabe sostener que los términos de género natural son, como los nombres propios, designadores rígidos, este rasgo de los términos de género natural no permite justificar la pke denomina a este tipo de enunciados "identificaciones 
tesis de que las identidades teóricas son enunciados que, en caso de ser verdaderos, son necesarios a posteriori. He de indicar de antemano que el carácter a posteriori de las identidades teóricas es un rasgo que voy a conceder, pues sin recurrir a la experiencia no cabe justificar la verdad de enunciados como los dos ejemplos de identidades teóricas mencionados. Pero voy a cuestionar la justificación ofrecida por Kripke de la necesidad que él atribuye a las identidades teóricas. Incluso si cabe aceptar que los términos de género natural son, como los nombres propios, designadores rígidos -cuestión a la que responderé de manera positiva-, alegaré que esta similitud entre ambos tipos de expresiones no permite justificar la tesis de que los enunciados de identidad verdaderos en los que figuran dos términos de género natural son, como aquéllos en los que figuran dos nombres propios, necesarios.

Conviene comenzar haciendo algunas observaciones acerca del marco en el que Kripke formula la tesis de que los términos de género natural son designadores rígidos. Es digno de mención que Kripke no presenta una caracterización muy precisa de los términos de género natural; él afirma que los términos de género natural son términos generales y que entre ellos se encuentran tanto términos contables, p.e., los términos "gato" y "tigre", como términos de masa, p.e., los términos "agua" y "oro" (Kripke, 1980, 134); justamente este último tipo de términos son los términos de sustancia. Si tomamos en consideración que Kripke emplea indistintamente las expresiones "términos de género natural" y "términos para géneros naturales", la conclusión es que los términos de género natural son caracterizados por Kripke como términos generales que designan - 0 , al menos, pretenden designar- géneros naturales. Esta caracterización sólo podrá adquirir perfiles más nítidos cuando se precise cómo se fija la referencia de los términos de género natural y cómo se conciben los géneros naturales.

En la primera conferencia de (1980) Kripke introduce el término "designador" como denominación común de los nombres propios y de las descripciones definidas, que son los dos tipos de términos singulares que Kripke toma en consideración. La definición de designador rígido se encuentra en las conferencias primera y segunda $y$, por tanto, aparece formulada antes de que Kripke atienda a los términos de género natural. Puesto que Kripke afirma que los términos de género natural son, como los nombres pro- pios, designadores rígidos, pero no presenta explícitamente una definición de designador rígido para los términos de género natural, habrá de suponerse que la caracterización de designador rígido aplicable a estos términos será similar a la propuesta para los términos singulares $y$, en especial, para los nombres propios.

La definición de designador rígido contenida en la primera edición de (1980) -publicada en 1972-, y en el escrito (1971), puede dar lugar a distintas caracterizaciones. Esta ambigüedad llevó a David Kaplan a dirigirse a Kripke para pedirle que clarificase dicha noción. En su respuesta Kripke afirma que la noción de designador rígido pretendida por él es la siguiente: "[U]n designador $d$ de un objeto $x$ es rígido si designa $x$ con respecto a todos los mundos posibles en los que $x$ existe y no designa un objeto distinto de $x$ con respecto a ningún mundo posible" (citado en Kaplan, 1989, 569). Esta definición de designador rígido deja abierta dos opciones. La primera es que un designador rígido designe el mismo objeto con respecto a todos los mundos posibles. La segunda opción es que un designador rígido designe el mismo objeto sólo con respecto a los mundos posibles en los que el objeto exista, careciendo de referencia con respecto a los demás mundos posibles. Siguiendo una terminología hoy usual, introducida inicialmente en Salmon (1981), los designadores rígidos que satisfacen la primera caracterización son designadores obstinados, mientras que los que satisfacen la segunda son designadores persistentes.

Ahora bien, aunque Kripke prefiere dejar abierta esa doble posibilidad para no verse involucrado en cuestiones planteadas por la posible no-existencia de un objeto, la caracterización de designador rígido aplicable a los nombres propios pretendida por Kripke es la primera de ellas, pues en el prefacio a (1980) Kripke nos indica que considera a los nombres propios como designadores rígidos de jure. Un designador es rígido de jure si al fijar su referencia se estipula que su referente es el mismo independientemente de que estemos hablando acerca del mundo actual o de un mundo posible distinto del actual. Puesto que Kripke considera a los nombres propios como rígidos de jure, él afirma que un nombre propio designa rígidamente su referente incluso con respecto a mundos posibles en los que el referente no exista.

Por el contrario, la mayoría de las descripciones definidas son designadores no-rígidos, es decir, pueden designar di- 
ferentes objetos con respecto a distintos mundos posibles, pues puede ocurrir que en distintos mundos posibles un objeto diferente sea el único que posea la propiedad expresada por la descripción. No obstante, Kripke reconoce que hay algunas descripciones definidas que son designadores rígidos, si bien no son designadores rígidos de jure, sino designadores rígidos de facto. En el caso de un designador rígido de facto no se estipula que hay un único objeto que constituye su referente con respecto a todo mundo posible, pero el predicado contenido en la descripción se aplica al mismo objeto con respecto a todos los mundos posibles 0 , al menos, con respecto a todos los mundos posibles en los que el objeto exista, dependiendo de si la descripción definida en cuestión es un designador obstinado o persistente.

Una consecuencia de la rigidez de los nombres propios es que los enunciados de identidad verdaderos en los que el signo de identidad es flanqueado por dos nombres propios son necesarios, es decir, verdaderos con respecto a todos los mundos posibles. Uno de los ejemplos más famosos es el enunciado de identidad "Héspero es Fósforo". Este enunciado es verdadero, puesto que los nombres "Héspero" y "Fósforo" designan el mismo objeto, es decir, el planeta Venus. Ahora bien, si esos nombres propios son, como Kripke pretende que son todos los nombres propios, rígidos de jure, dichos nombres designarán el planeta Venus con respecto a todos los mundos posibles, a consecuencia de lo cual el enunciado "Héspero es Fósforo" es necesario. Pero, por otra parte, el que esos dos nombres propios designan el mismo objeto fue el resultado de un descubrimiento empírico y es algo que no podiamos saber a priori. Por tanto, el enunciado "Héspero es Fósforo" es, aunque necesario, verdadero a posteriori.

Llegados a este punto cabe plantear dos cuestiones de las que tendremos que ocuparnos. En primer lugar, si la caracterización de designación rígida para los términos de género natural y la concepción de Kripke acerca de los géneros naturales permiten sostener que los términos de género natural son designadores rígidos. En segundo lugar, y supuesta una respuesta positiva a la primera cuestión, si de la rigidez de los términos de género natural se sigue que las identidades teóricas son, como los enunciados de identidad en los que figuran dos nombres propios, enunciados necesarios, en caso de ser verdaderos -como ya indicamos, no vamos a cuestionar su carácter a posteriori-.
No obstante, antes de ocuparnos de la primera cuestión es pertinente mencionar otras dos similitudes que existen, según Kripke, entre los términos de género natural y los nombres propios: la no-descripcionalidad y el carácter histórico-causal de la fijación de su referencia. Los términos de género natural son, como los nombres propios, no-descripcionales, es decir, no son sinónimos de descripciones usualmente asociadas con ellos por los hablantes y que determinarían su referencia. Ahora bien, si la fijación de la referencia de los nombres propios y de los términos de género natural no es explicada de esa manera, es decir, mediante descripciones de las que son sinónimos, ha de proponerse una explicación alternativa acerca de cómo se fija su referencia. A este respecto Kripke propone una teoria histórico-causal, tanto para los nombres propios como para los términos de género natural. En el caso de los términos de género natural Kripke sostiene que estos términos son introducidos mediante ostensión en presencia de entidades paradigmáticas del género o por medio de descripciones que expresan propiedades, usualmente contingentes, de dichas entidades; en ambos casos la extensión de un término de género natural comprenderá todas las entidades del mismo género que las involucradas en la introducción del término, y la relación de identidad de género viene constituida por propiedades subyacentes de dichas entidades, cuyo descubrimiento es objeto de la investigación empírica. Una vez que el término de género natural ha sido introducido, es transmitido a otros hablantes mediante cadenas causales de comunicación y estos hablantes usarán el término para referirse a entidades del género, aunque las descripciones que asocien con el término no expresen propiedades identificadoras del género.

La no-descripcionalidad y el carácter histórico-causal de la fijación de su referencia son rasgos que usualmente aparecen vinculados con la afirmación de que los nombres propios y los términos de género natural son designadores rígidos. Pero por lo que concierne a los términos de género natural dichos rasgos no son suficientes para justificar la tesis de que son designadores rígidos. La justificación de esta tesis dependerá principalmente de cómo se caracterice la noción de designador rígido para los términos de género natural y de cómo sean concebidos los géneros naturales, es decir, los referentes o designata de los términos de género natural.

Por lo que respecta a esta primera cuestión, conviene volver a tomar en consideración la caracterización de de- 
signador rígido para los términos singulares pretendida por Kripke. Puesto que en la tercera conferencia de (1980) él extiende la noción de designador rígido a los términos de género natural, habremos de extender esa caracterización de designación rígida a los términos de género natural 0 , en general, a los términos de género. A este respecto una extensión natural, y la única que tomaré en consideración, es la siguiente: un designador " $d$ " de un género $g$ es rígido si y sólo si designa $g$ con respecto a todos los mundos posibles en los que $g$ existe y no designa un género distinto de $g$ con respecto a ningún mundo posible? .

En lo tocante a la segunda cuestión, ha de señalarse que Kripke concibe los géneros naturales como entidades abstractas instanciadas en entidades concretas. Así afirma:

[E]n general, la referencia de los términos de género natural (p.e., de géneros animales, vegetales y químicos) se fija de esta manera: la sustancia es definida como el género instanciado por (casi la totalidad) de una muestra dada (Kripke, $1980,135-136)^{2}$.

La concepción de los géneros naturales como ciertos universales o entidades abstractas, instanciadas en entidades concretas, es la posición predominante hoy en día entre los autores que aceptan la tesis de que los términos de género natural son designadores rígidos. Esta concepción permite sostener que los términos de género natural son designadores rígidos, de acuerdo con la caracterización de designación rígida para estos términos $y$, en general, para los términos de género propuesta anteriormente, pues las entidades abstractas designadas por los términos de género natural serán las mismas con respecto a todos los mundos posibles 0 , al menos, con respecto a los mundos posibles en los que dichas entidades existan. Ahora bien, la concepción de los referentes de los términos de género natural como entidades abstractas instanciadas en entidades concretas es aplicable a los referentes de todos los términos generales que se aplican a entidades concretas, lo que nos llevará a aceptar que no sólo los términos de género natural, sino muchos otros términos generales, son designadores rígidos.

Llegados a este punto conviene introducir ciertas precisiones. Como ya indicamos, Kripke sostiene que los nombres propios, es decir, términos singulares semánticamente simples son designadores rígidos, mientras que la mayoría de las descripciones definidas, es decir, términos singulares que poseen una estructura semánticamente relevante, son designadores no-rígidos. Una vez que se ha concedido que la concepción de los referentes de los términos generales como tales entidades abstractas permite sostener que los términos de género natural y muchos otros términos generales son designadores rígidos, cabe afirmar que los términos generales que sean semánticamente simples serán designadores rígidos; así, por ejemplo, los términos "agua" y "oro" serán designadores rígidos, pero también lo serán otros términos como "soltero" y "mesa". De este modo no sólo términos generales no-descripcionales, como, según Kripke, son los términos de género natural, sino también términos descripcionales paradigmáticos, como el término "soltero", serán designadores rígidos. Una diferencia entre estos últimos y los términos de género natural radicará en el otro rasgo ya mencionado, a saber, en el carácter histórico-causal de la fijación de la referencia de los términos de género natural.

Sin embargo, de acuerdo con dicha concepción de los referentes de los términos de género natural $y$, en general, de los términos generales, no todos los términos generales serán designadores rígidos. Más precisamente, la mayoría de los términos generales que poseen una estructura semánticamente relevante, es decir, la mayoría de los términos generales semánticamente compuestos no serán designadores rígidos. Así, por ejemplo, los términos "líquido empleado para lavarse las manos" y "metal favorito de Juan" no serán designadores rígidos, pues podrán designar con respecto a distintos mundos posibles distintos líquidos o metales, concebidos éstos como entidades abstractas. No obstante, habrá algunos términos generales semánticamente compuestos que sí serán designadores rígidos, como, por ejemplo, los términos "sustancia compuesta de moléculas que constan de dos átomos de hidrógeno y de un átomo de oxígeno" y "elemento con el número atómico 79". Y llegados a este punto cabe extender la similitud entre la rigidez de los términos singulares y de los términos generales para concluir que los términos generales semánticamente simples son, como los nombres propios, rígidos de jure, mientras que los términos semánticamente compuestos, ya sean singulares o generales, que son rígidos son sólo rígidos de facto.

Ahora bien, de acuerdo con esta conclusión los dos ejemplos de identidades teóricas mencionados contienen un 
designador rígido de jure y uno de facto, y mientras que los designadores del primer tipo son obstinados, los del segundo tipo pueden ser tanto obstinados como persistentes. Ésta es una razón por la que no cabe establecer la necesidad de las identidades teóricas siguiendo el mismo proceder que el mencionado con respecto a los enunciados de identidad en los que figuran dos nombres propios $y$, por tanto, dos designadores obstinados. A este respecto, sin embargo, conviene señalar que, al menos en una ocasión, Kripke acepta un sentido débil de necesidad: "[p]odemos considerar los enunciados como necesarios si cuando quiera que los objetos mencionados en ellos existan, el enunciado sería verdadero" (Kripke, 1971, 137)³.

No obstante, incluso aceptando que los términos de género natural son designadores rígidos -obstinados 0 persistentes-, voy a cuestionar que de la rigidez de los términos de género natural se siga que las identidades teóricas son enunciados que, en caso de ser verdaderos, son necesarios, ya se entienda la necesidad en sentido estricto o en sentido débil. A este respecto tomaré en consideración los dos ejemplos de identidades teóricas mencionados, a saber, los enunciados " $\mathrm{El}$ agua es $\mathrm{H}_{2} \mathrm{O}$ " y "El oro es el elemento con el número atómico 79", centrándome en el primero de ellos, pues similares consideraciones se aplicarian al segundo.

Conviene señalar que estos enunciados pueden ser interpretados como enunciados de identidad, pero también como bicondicionales cuantificados universalmente. Interpretado de esta última manera el enunciado "El agua es $\mathrm{H}_{2} \mathrm{O}$ " es verdadero si y sólo si para toda porción (del mundo actual) se cumple que una porción instancia la sustancia agua si y sólo si instancia la sustancia $\mathrm{H}_{2} \mathrm{O}$ y, por tanto, si y sólo si los términos generales "agua" y " $\mathrm{H}_{2} \mathrm{O}$ " tienen la misma extensión, es decir, se aplican a los mismos objetos". De esta manera el enunciado "El agua es $\mathrm{H}_{2} \mathrm{O}$ " es verdadero si y sólo si sus términos componentes son coextensivos, pero de aquí no se sigue ni que sean necesariamente coextensivos ni que ese enunciado sea, supuesta su verdad, necesario.

Obtenemos la misma conclusión si consideramos el enunciado "El agua es $\mathrm{H}_{2} \mathrm{O}$ " como un enunciado de identidad, en el que el signo de identidad (de segundo orden) es flanqueado por los términos generales "agua" y " $\mathrm{H}_{2} \mathrm{O}$ ". Este enunciado será verdadero si y sólo si la sustancia agua y la sustancia $\mathrm{H}_{2} \mathrm{O}$ son idénticas en el mundo actual, pero la condición necesaria y suficiente para la identidad de esas sustancias en el mundo actual es que sus instancias en el mundo actual sean las mismas, es decir, que los términos "agua" y " $\mathrm{H}_{2} \mathrm{O}$ " sean coextensivos. Dicho de manera general, las identidades teóricas, consideradas como enunciados de identidad en los que figuran dos términos de género natural, son enunciados verdaderos si y sólo si los géneros designados por sus dos designadores rígidos componentes son idénticos en el mundo actual, pero de aquí no se sigue la identidad de ambos géneros en todos los mundos posibles, es decir, no se sigue que sus instancias sean las mismas en todos los mundos posibles $y_{\text {, por }}$ tanto, no se sigue que esos enunciados, en caso de ser verdaderos, sean necesarios.

Kripke pretende justificar la necesidad de ciertos enunciados (verdaderos) de identidad, tanto en el caso de aquéllos en los que figuran dos nombres propios como en el caso de las identidades teóricas entendidas como enunciados de identidad, apelando a que la referencia de los términos componentes de dichos enunciados es la misma en el mundo actual y a que dichos términos son designadores rígidos. Es así como se justificaba la necesidad del enunciado "Héspero es Fósforo". La justificación pretendida por Kripke de la necesidad de las identidades teóricas (verdaderas) procede en principio de la misma manera -véase el primer texto de Kripke citado en el presente artículo-, pero, como hemos indicado, este proceder, por sí solo, es insuficiente para obtener la conclusión deseada. Por este motivo, no es de extrañar que Kripke se vea movido a complementarlo con consideraciones adicionales.

A la hora de justificar que identidades teóricas como "El agua es $\mathrm{H}_{2} \mathrm{O}$ " y "El oro es el elemento con el número atómico 79" son enunciados necesarios, Kripke apela adicionalmente a consideraciones a priori acerca de en qué consiste la esencia de un género natural (o de sus instancias) y a este respecto Kripke afirma que la esencia de una sustancia se identifica con su estructura atómica (Kripke, 1980, 124-125, 128). De esta manera si el agua es esencialmente la sustancia compuesta de moléculas que constan de dos átomos de hidrógeno y de un átomo de oxígeno -abreviada como " $\mathrm{H}_{2} \mathrm{O}$ "-, entonces ser $\mathrm{H}_{2} \mathrm{O}$ es la esencia del agua y la identidad teórica correspondiente será, en caso de ser verdadera, necesaria. Pero conviene subrayar que esas pretensiones sobre la esencia de las sus- 
tancias vienen justificadas, según Kripke, por un "análisis filosófico a priori" (Kripke, 1971, 153). Él afirma:

Todos los casos de necesidad a posteriori defendidos en el texto tienen el carácter especial atribuido a los enunciados matemáticos: el análisis filosófico nos dice que no pueden ser contingentemente verdaderos, por lo que cualquier conocimiento empírico de su verdad es automáticamente conocimiento empírico de que son necesarios. Esta caracterización se aplica, en especial, a los casos de enunciados de identidad y de esencia (Kripke, 1980, 159).

Sin embargo, ni el análisis filosófico de Kripke ni sus conclusiones requieren ser compartidas. El término "agua" puede ser considerado como un designador rígido, cuya referencia es una sustancia, y las instancias de esa sustancia y de la sustancia $\mathrm{H}_{2} \mathrm{O}$ son idénticas en el mundo actual. Por tanto, en este sentido el enunciado "El agua es $\mathrm{H}_{2} \mathrm{O}$ " es verdadero. No obstante, de aquí no se sigue que ese enunciado sea necesario; para obtener esta conclusión ha de aceptarse una tesis que cabe ser cuestionada, a saber, que la esencia de una sustancia se identifique con su estructura atómica $y$, por tanto, que es necesario que las sustancias o más bien sus instancias hayan de tener en todos los mundos posibles en los que existan la estructura atómica que tienen en el mundo actual. Kripke sostendria que la verdad de esta tesis viene establecida por un análisis filosófico a priori, pero llegados a este punto la discusión filosófica se encuentra en un punto muerto.

De esta manera la designación rígida y la identidad de referencia en el mundo actual, que permiten justificar que los enunciados de identidad en los que figuran dos nombres propios son, en caso de ser verdaderos, necesarios, no permite justificar la tesis correspondiente en relación con los términos de género natural. El término "agua", en su uso en la ciencia ${ }^{5}$, puede ser considerado como un designador rígido de la sustancia cuyas instancias son porciones de agua, y el término " $\mathrm{H}_{2} \mathrm{O}$ " puede ser considerado como un designador rígido cuyas instancias son (compuestos de) moléculas que constan de dos átomos de hidrógeno y de un átomo de oxígeno. Las instancias de ambas sustancias son idénticas en el mundo actual, pero Kripke no ha establecido que sean necesariamente idénticas.

La conclusión de las consideraciones precedentes es que, aunque cabe sostener que los términos de género natural son designadores rígidos, el carácter rígido de estos términos no permite, por sí solo, justificar la tesis de que las identidades teóricas son enunciados que, en caso de ser verdaderos, son necesarios ${ }^{6}$.

\section{NOTAS}

1 Las afirmaciones acerca de la existencia de un género con respecto a un mundo posible dependerán de las concepciones sobre los géneros y sobre las relaciones entre los géneros y las entidades del género que se sostengan, pero en lo siguiente voy a mantenerme en lo posible neutral con respecto a estas cuestiones, limitándome a asumir los escasos compromisos que Kripke parece haber adoptado al respecto.

2 La puntualización contenida en este texto -"casi la totalidad"-, que en lo siguiente dejaré de lado, se debe a que entre las entidades involucradas en la fijación de la referencia de un término de género natural pueden encontrarse un pequeño número de entidades de un género distinto.

3 Cabe formar tanto con designadores persistentes como con designadores obstinados un tipo de enunciados (condicionales) que son necesarios en sentido estricto, es decir, verdaderos con respecto a todos los mundos posibles. Supongamos que "A" y "B" son designadores rígidos de la misma entidad, independientemente de que sean designadores obstinados o persistentes; en este caso el enunciado condicional que contiene como antecedente el enunciado que afirma la existencia de tal entidad y

Aceptado: 3 de enero de 2010 
como consecuente el enunciado de identidad correspondiente será un enunciado necesario; por tanto, bajo dicho supuesto, los enunciados de la forma "Si A existe, entonces $A$ es idéntico a $B$ " son necesarios.

4 El término ${ }^{~} \mathrm{H}_{2} \mathrm{O}$ " es, en sentido estricto, una descripción definida, a saber, la descripción "La sustancia compuesta de moléculas que constan de dos átomos de hidrógeno y de un átomo de oxígeno" (o una descripción equivalente a ésta). Cuando me refiero al término " $\mathrm{H}_{2} \mathrm{O}$ " como un término general estoy entendiendo por tal el término general con el que se ha formado la descripción, es decir, el término obtenido a partir de la descripción eliminando el artículo "la".

5 Cabe cuestionar que el término "agua" en su uso cotidiano sea un designador rígido, pues su referencia no viene de- terminada exclusivamente mediante una propiedad subyacente, como la propiedad de ser (o estar compuesto de) $\mathrm{H}_{2} \mathrm{O}$. Factores como las propiedades macroscópicas y funcionales normales del agua, así como el contexto y los intereses de los hablantes pueden desempeñar un papel en la determinación de la extensión del término "agua" en el lenguaje cotidiano y en la correcta aplicación de este término en una ocasión dada.

6 La elaboración de este artículo ha contado con la financiación del proyecto HUM2005-03439 del Ministerio de Educación y Ciencia, así como con la concedida por la Consejería de Educación de la CAM y la Universidad Complutense de Madrid al grupo de investigación 930174 ("Filosofía del lenguaje, de la naturaleza y de la ciencia").

\section{BIBLIOGRAFÍA}

Kaplan, David (1989): "Afterthoughts", en Almog, Joseph et al. (eds.), Themes from Kaplan, Nueva York: Oxford University Press, pp. 565-614.

Kripke, Saul (1971): "Identity and necessity", en Munitz, Milton (ed.), Identity and Individuation, Nueva York: New York University Press, pp. 135-164.

Kripke, Saul (1980): Naming and Necessity, Oxford: Blackwell. Reimp. revisada y con prefacio añadido de "Naming and necessity", en Davidson, Donald y Gilbert Harman (eds.), Semantics of Natural Language, Dordrecht: Reidel, 1972.

Salmon, Nathan (1981): Reference and Essence, Princeton: Princeton University Press. 